\title{
Farmacocinética y farmacodinamia de antimicrobianos: a propósito de pacientes con neutropenia y fiebre
}

\author{
Javier R. Garzón, Sonia Cuervo M., Julio Gómez R. y Jorge A. Cortés
}

\section{Pharmacokinetics and pharmacodynamics of antibacterial agents: on account of febrile neutropenic patients}

Febrile neutropenia is a serious complication of antineoplastic therapy and it is more commonly found in hematologic patients, associated with high mortality rates. Inadequate tissue concentration of antimicrobials has been described as a cause of therapeutic failure which also has been related to a low interstitial concentration for hydrophilic antibiotics. In critically ill patients it may occur an accumulation of compartmental fluids which can be related to an increase in the distribution volume or changes in clearance of antimicrobials. Pharmacokinetic and pharmacodynamic parameters of antimicrobials are reviewed, which can be used as a tool to optimize the efficacy of antimicrobial therapy in order to avoid failures and resistance selection.

Key words: Anti-infective agents/administration \& dosage/therapeutic use, drug therapy/standards, fever/drug therapy/etiology, humans, hematologic neoplasms /complications, neutropenia/drug therapy/etiology.

Palabras clave: Agentes antibacterianos/administración \& dosificación/uso terapéutico, terapéutica, fiebre / etiología/terapia, humanos, neoplasias hematológicas/complicaciones, neutropenia/etiología/terapia.

\section{Introducción}

$\mathrm{E}$ n los pacientes con cáncer sometidos a quimioterapia, que desarrollan neutropenia y fiebre (de ahora en adelante neutropenia febril), la mitad tiene una infección establecida u oculta con mortalidad de hasta $60 \%{ }^{1}$, razón por la cual desde los años sesenta se estableció la terapia empírica con antimicrobianos de amplio espectro, con el objetivo de erradicar principalmente bacterias gramnegativas. Con la terapia empírica, se ha logrado disminuir la mortalidad asociada con esta complicación aproximadamente entre 7 y 39\%, según diferentes series ${ }^{2-5}$ en adultos y en 0,6 a 7,5 \% en niños ${ }^{6-9}$, pero a pesar de esta disminución la mortalidad sigue siendo elevada.

Entre los factores que pueden explicar la elevada mortalidad, están la demora en el inicio del tratamiento, la resistencia bacteriana, las infecciones fúngicas invasoras y las concentraciones inapropiadas de los antimicrobia$\operatorname{nos}^{1-3,5}$.

En los pacientes críticamente enfermos ocurren variaciones en el volumen extracelular, que pueden causar alteraciones en las concentraciones séricas o tisulares de los medicamentos ${ }^{10}$. Tanto en pacientes neutropénicos, como en modelos animales se ha reportado disminución en las concentraciones séricas, tisulares y en líquidos corporales de los agentes anti-infecciosos, lo cual se puede asociar con inadecuados niveles terapéuticos y estos a su vez pueden causar fracasos y seleccionar cepas resistentes.

Los parámetros farmacocinéticos y farmacodinámicos (conocidos con alguna frecuencia como PK/PD por las siglas en inglés de pharmacokinetics / pharmacodynamics) pueden permitir a los médicos clínicos optimizar el modo de administración de los antimicrobianos en pacientes con neutropenia febril aumentando potencialmente su eficacia. Dicho de otra forma, la adecuada dosificación de la terapia antimicrobiana puede llevar a mejorar los desenlaces de los pacientes con neutropenia febril ${ }^{11}$. El objetivo de la presente revisión es mostrar el impacto de los parámetros $\mathrm{PK} / \mathrm{PD}$ en pacientes con neutropenia febril.

\section{Aspectos microbiológicos en América Latina}

Los patrones epidemiológicos de las infecciones bacterianas en los pacientes con neutropenia presentan cambios periódicos y están influenciados por varios factores ${ }^{12}$, incluyendo la gravedad y la duración de la neutropenia, la naturaleza e intensidad de la terapia anti-neoplásica, los factores relacionados con el hospedero, la presión selectiva creada por el uso de antibacterianos profilácticos o terapia antimicrobiana empírica, el uso de catéteres centrales y otros dispositivos médicos externos, factores ambientales, geográficos y la duración de la estancia hospitalaria. Sin embargo, uno de los determinantes más importantes es la distribución local de microorganismos en estos grupos de pacientes, información que ha sido limitada en nuestro escenario latinoamericano.
Universidad Nacional de Colombia, Bogotá, Colombia. Facultad de Medicina (JRG, SCM, JAC).

Instituto Nacional de Cancerología, Bogotá, Colombia (SCM, JGR).

Los autores declaran no tener conflictos de interés. Fuente de financiamiento: jornadas docentes, profesores Facultad de Medicina, Universidad Nacional de Colombia.

Recibido: 25 de noviembre de 2010 Aceptado: 27 de septiembre de 2011

Correspondencia a:

Javier R. Garzón garzonjavier@yahoo.com 
En la década de los 50, Staphylococcus aureus era el responsable de la mayor parte de las infecciones en pacientes neutropénicos. En las décadas de los 60 y 70, la mayor parte de las infecciones eran debidas predominantemente a bacilos gramnegativos, especialmente Pseudomonas aeruginosa, Klebsiella pneumoniae y Escherichia coli ${ }^{13}$ las cuales se asociaban a elevada mortalidad (superior a 50\%), de no ser tratadas dentro de las primeras 48 horas. Estos hallazgos llevaron a la utilización de la terapia empírica, lo cual ha causado una dramática disminución en la tasa de mortalidad de los pacientes neutropénicos con cáncer ${ }^{14}$.

En la década de los 80 se presentó un nuevo cambio en el tipo de bacterias que causan infección en los pacientes con neutropenia y cáncer, probablemente como consecuencia de los factores previamente mencionados, que llevó al predominio de las cocáceas grampositivas. En los años 90 se observó un aumento de infecciones graves por bacterias del grupo viridans en pacientes neutropénicos con leucemia aguda y sometidos a trasplante de precursores hematopoyéticos o tratamiento poliquimioterápico ${ }^{13}$. En la mayoría de centros, aproximadamente $70 \%$ de los aislados en sangre eran cocáceas grampositivas ${ }^{15}$.

Sin embargo, aunque los microorganismos grampositivos causaban bacteriemia más frecuentemente que los gramnegativos, se encontró predominancia de estos últimos en la mayoría de los otros sitios de infección. Adicionalmente, se duplicó la frecuencia de infecciones polimicrobianas desde 1970, encontrándose responsables de 23 al 31\% de las infecciones bacterianas ${ }^{16}$.

En los últimos años se han incrementado nuevamente las infecciones por bacilos gramnegativos; existen reportes de la aparición de este fenómeno tanto en instituciones donde se emplea ciprofloxacina como profilaxis como en las que no se utiliza ${ }^{5}$. En América Latina disponemos de limitada información al respecto. En el año 2003 en el Instituto Nacional de Cancerología, en Colombia, se vigilaron 128 pacientes con diagnóstico de neutropenia febril que recibieron antimicrobianos. Treinta y ocho por ciento fueron niños. Se obtuvieron 83 cultivos potencialmente invasores de 45 pacientes (35\%), 52\% correspondieron a cocáceas grampositivas, $36 \%$ a bacilos gramnegativos y $12 \%$ a levaduras y micelios. Staphylococcus aureus fue el microorganismo más frecuente, seguido de E. coli, $S$. epidermidis, Enterococcus faecalis y K. pneumoniae. Se aislaron especies de Streptococcus en $9 \%$ de los casos ${ }^{17}$. Entre enero de 1998 y diciembre de 2003 en el Hospital Universitario de Caracas, en Venezuela, se registraron 576 episodios de neutropenia febril. Se reportó algún tipo de aislado en $41 \%$ de 940 cultivos. Predominaron los bacilos gramnegativos (48\%), seguidos por cocáceas grampositivas $(35,1 \%)$, hongos $(11,5 \%)$ y otros agentes $(5,4 \%)$. Individualmente, Staphylococcus coagulasa negativa $(22,4 \%)$ y E. coli $(13,4 \%)$ fueron los más frecuentes ${ }^{18}$.
En el Hospital San Vicente de Paul de Medellín, Colombia, se estudiaron en forma retrospectiva 441 historias clínicas en el período comprendido entre enero de 2003 y diciembre de 2005. Se identificaron las características de 117 episodios de neutropenia febril en 96 pacientes de los cuales se obtuvo aislado microbiológico en $51 \%$ de los casos, siendo $59 \%$ bacilos gramnegativos y $32 \%$ cocáceas grampositivas ${ }^{4}$. Entre enero de 2004 y agosto de 2007 en el Hospital Clínico Universidad Católica en Santiago de Chile se registraron 154 episodios de neutropenia febril; de estos, $30,5 \%$ tuvieron hemocultivos positivos y $51 \%$ correspondieron a bacilos gramnegativos, $41 \%$ a cocáceas grampositivas y $8 \%$ a levaduras ${ }^{19}$. En un segundo estudio, más reciente, en el 2007, se registraron 214 episodios de neutropenia febril en el Instituto Nacional de Cancerología en Bogotá, Colombia, de los cuales se aislaron microorganismos en $43,5 \%$. La mayoría de los aislados provenían de sangre (75\%); predominaron los bacilos gramnegativos $(58 \%)$, las cocáceas grampositivas fueron $36 \%$, los hongos fueron el $6 \%$ de los aislados ${ }^{20}$.

A manera de resumen, en la Tabla 1 se presenta la distribución general de los microorganismos identificados en pacientes con neutropenia febril post-quimioterapia en América Latina.

En las décadas de los 80 y 90 la mortalidad de los pacientes con neutropenia febril se encontraba entre $26 \mathrm{y}$ $39 \% \%^{2-4}$; sin embargo, en los últimos años se ha reportado disminución de hasta $7 \%$ en lo cual muy probablemente la terapia empírica con antimicrobianos de amplio espectro ha tenido un papel importante 5 .

\section{Tratamiento antimicrobiano de los pacientes con neutropenia febril post-quimioterapia}

Los pacientes con cáncer tratados con quimioterapia presentan múltiples factores que predisponen a infecciones por microorganismos como $P$. aeruginosa (corticosteroides, desnutrición, uso de antibacterianos de amplio espectro); enterobacterias (disrupción de barreras mucosas, co-morbilidades, uso de antibacterianos de amplio espectro); S. aureus (uso de dispositivos intravasculares, co-morbilidades, terapia antimicrobiana previa); Streptococcus grupo viridans (disrupción de barreras mucosas); S. pneumoniae (hipogamaglobulinemia, asplenia funcional), Candida spp (disrupción de barreras

Tabla 1. Frecuencia de aislados microbiológicos en pacientes con neutropenia febril en América Latina

\begin{tabular}{|lc|}
\hline Tipo de microorganismo & Frecuencia (\%) \\
\hline Bacilos gramnegativos & $48-59^{4,13,14,15,16}$ \\
\hline Cocáceas grampositivas & $32-52^{4,13,14,15,16}$ \\
\hline Hongos & $6-11^{4,13,14,15,16}$ \\
\hline
\end{tabular}


mucosas, neutropenia); e infecciones por microorganismos intracelulares (corticosteroides, linfomas) ${ }^{21}$.

En la década de los 70 se encontraron tasas de respuesta al tratamiento antimicrobiano de 60 a $70 \%$ con la combinación de un $\beta$-lactámico con actividad antipseudomonas y un aminoglucósido. Durante la década de los 80 la aparición de nuevos antibacterianos con mayor espectro (cefalosporinas de tercera y cuarta generación, carbapenems, fluoroquinolonas y $\beta$-lactámicos con inhibidor de $\beta$-lactamasa) llevaron a la introducción de la monoterapia que es más fácil de administrar y menos tóxica ${ }^{14}$. Varias guías internacionales ${ }^{1,21-23}$ recomiendan tratamiento en monoterapia con ceftazidima, cefepime, piperacilina/ tazobactam, imipenem o meropenem. Algunas de estas guías plantean la opción de adicionar un aminoglucósido o ciprofloxacina como terapia combinada; sin embargo, dos meta-análisis ${ }^{24,25}$ evaluaron la utilidad de la terapia combinada con un aminoglucósido en los que no se encontraron ventajas con la combinación como terapia empírica de pacientes con neutropenia febril; en cambio se encontró una mayor frecuencia de efectos adversos, principalmente nefrotoxicidad.

\section{Cambios farmacocinéticos en los pacientes críticamente enfermos}

En los pacientes con sepsis, trauma, hipoalbuminemia, nutrición parenteral, líquidos endovenosos, insuficiencia cardiaca, quemados o con acumulación compartimental de líquidos, se ha descrito un aumento en los volúmenes de distribución de los medicamentos. En pacientes con fases tardías de quemaduras, leucemia aguda y la fase hiperdinámica de la sepsis se ha reportado aumento en la depuración de medicamentos. En contraposición, en pacientes con insuficiencia renal y los mayores de 75 años se ha encontrado disminución de la depuración de medicamentos ${ }^{10}$.

Hasta en 54\% de los pacientes críticamente enfermos se ha encontrado disfunción hepática, asociada a factores como hipotermia, sepsis e hipotensión, lo cual puede causar disminución de la depuración de los medicamentos eliminados por esta vía, debido a disminución en la actividad enzimática hepatocelular, disminución del flujo sanguíneo y/o biliar ${ }^{26,27}$.

Las variaciones que ocurren en el líquido extracelular y/o en la función renal o hepática son los mecanismos fisiopatológicos más frecuentes y relevantes que pueden afectar la disposición de los medicamentos en los pacientes críticamente enfermos ${ }^{27}$.

Los antimicrobianos hidrofílicos $\beta$-lactámicos, aminoglucósidos y glucopéptidos) al igual que los moderadamente lipofílicos (ciprofloxacina, gatifloxacina y levofloxacina) conllevan el mayor riesgo de presentar fluctuaciones diarias en las concentraciones plasmáticas que pueden requerir ajustes de dosis. De hecho, los fár- macos hidrofílicos exhiben un volumen de distribución limitado al espacio extracelular y sus concentraciones plasmáticas e intersticiales pueden disminuir notablemente con la formación de tercer espacio. La presencia de un estado edematoso, independiente del mecanismo fisiopatológico subyacente, juega un papel importante dado que altera la distribución de los fármacos; por lo tanto, se debe considerar el uso de mayores dosis para la mayoría de los antimicrobianos hidrofílicos en busca de asegurar concentraciones terapéuticas adecadas ${ }^{28}$.

\section{Farmacocinética y farmacodinamia}

Hasta la fecha, las consideraciones acerca de terapia antimicrobiana en los pacientes con neutropenia febril se han enfocado principalmente en determinar los manejos de primera y segunda línea, el uso de monoterapia vs terapia combinada, el papel de los glucopéptidos y la identificación de pacientes de alto y bajo riesgo, sin considerar el comportamiento farmacocinético y farmacodinámico de los agentes antibacterianos recomendados que usualmente se ha encontrado alterado tanto en los pacientes con neutropenia como en modelos de animales neutropénicos ${ }^{11}$.

La cantidad de antimicrobiano que llega a la bacteria y su sitio activo depende de la concentración en el líquido intersticial, la cual es proporcional a la concentración sérica; en consecuencia, esta concentración sérica del antimicrobiano se correlaciona con la erradicación bacteriana. Para que un antibacteriano pueda erradicar un microorganismo debe unirse a su sitio activo en la bacteria, lograr una concentración adecuada y permanecer durante el tiempo suficiente en el mismo ${ }^{29}$.

Tradicionalmente se han utilizado la concentración inhibitoria mínima (CIM) y la concentración bactericida mínima (CBM) como predictores de la potencia de la interacción antibacteriano-microorganismo; sin embargo, estos parámetros no brindan información sobre el comportamiento en el tiempo de la actividad antimicrobiana ni de los efectos post antimicrobiano ${ }^{30}$.

La apropiada dosificación de los antimicrobianos es clave para la erradicación de las bacterias causantes de infección y un factor importante en la emergencia y proliferación de cepas resistentes. La óptima dosificación depende del entendimiento de la actividad microbiológica del antimicrobiano en cuestión, la susceptibilidad de la bacteria y la farmacocinética del antimicrobiano (que puede verse afectadas por factores relacionados al paciente). Estos factores determinan las propiedades farmacodinámicas del antimicrobiano (habilidad para erradicar las bacterias del sitio de infección) ${ }^{32}$.

De esta manera, la farmacología de la antibioterapia se puede dividir en dos componentes: la primera es la farmacocinética que hace referencia a la absorción, distribución y eliminación de los medicamentos (estos factores 
combinados con el régimen de dosificación determina el comportamiento en el tiempo de las concentraciones de los medicamentos en tejidos y líquidos corporales); la segunda es la farmacodinamia que consiste en la relación entre las concentraciones séricas y los efectos farmacológicos o tóxicos de los medicamentos. Con respecto a los antimicrobianos, el principal interés es la relación entre la concentración y el efecto antimicrobiano. El comportamiento en el tiempo de la actividad antimicrobiana es el reflejo de la relación entre la farmacocinética $(\mathrm{PK})$ y la farmacodinamia $(\mathrm{PD})^{30}$.

La identificación de los parámetros $\mathrm{PK} / \mathrm{PD}$ que mejor se correlacionan con eficacia se dificulta por el grado de interdependencia que hay entre los mismos; por ejemplo: una dosis elevada de un antimicrobiano produce una concentración máxima sobre CIM alta, un área bajo la curva elevada y mayor duración de tiempo sobre CIM. De esta manera, si una dosis más alta de un antimicrobiano produce un mejor efecto terapéutico, es difícil diferenciar qué parámetro tiene mejor relación con dicho efecto. Buscando reducir la interdependencia entre estos parámetros se han realizado estudios que incluyen diferentes intervalos de dosificación, los cuales se conocen como estudios de fraccionamiento de dosis. Dividiendo dosis totales en 1, 2, 4, 8, ó 24 dosis en intervalos cada $24,12,6,3$, y 1 hora, se ha buscado aclarar qué parámetro se correlaciona mejor con eficacia in vivo ${ }^{31}$. Basados en estos estudios se han encontrado tres parámetros $\mathrm{PK} / \mathrm{PD}$ que pueden predecir la eficacia de los antibacterianos:

Concentración máxima sobre CIM $\left(C_{\max } / C I M\right)$ : para antimicrobianos como los aminoglucósidos, la actividad bactericida es máxima en el momento de mayores concentraciones séricas (Cmax); según las concentraciones disminuyen también lo hace la actividad bactericida. Sin embargo, cuando las concentraciones séricas de los antimicrobianos dependientes de concentración caen por debajo de la CIM puede haber supresión persistente del crecimiento bacteriano gracias a los efectos postantibiótico, y la duración de los mismos depende a su vez de la concentración en suero de los antimicrobianos (a mayor concentración sérica mayor es la duración del efecto post-antibiótico y menor es la población bacteriana para el momento de la siguiente dosis).

Área bajo la curva sobre CIM (ABC/CIM): para antimicrobianos como las quinolonas, azitromicina, glucopéptidos, tigeciclina y linezolid, la máxima eficacia depende de la magnitud y la duración de la exposición. El ABC depende tanto del volumen de distribución como del aclaramiento. Los antibacterianos que presentan este comportamiento, también se caracterizan por presentar efectos post antibiótico prolongados.
Tiempo sobre CIM $(T>C I M)$ : los agentes bactericidas dependientes de tiempo como los $\beta$-lactámicos, dependen del tiempo que permanecen sobre la CIM para lograr su efecto antimicrobiano, es decir, tienen actividad bactericida relativamente lenta con un leve aumento cuando las concentraciones se encuentran por encima de cierto punto que es aproximadamente 4 a 5 veces la CIM; no obstante, su actividad no aumenta con concentraciones mayores. Estos medicamentos tienen efectos post-antibiótico cortos contra las bacterias grampositivas y prácticamente ningún efecto post- antibiótico contra las bacterias gramnegativas, a excepción de los carbapenémicos. Por estas razones se debería usar dosificación más frecuente, infusiones continuas o prolongadas para asegurar que las concentraciones se mantengan por encima de la CIM la mayor parte del intervalo de dosificación ${ }^{33}$.

Se debe tener presente que, por las razones previamente mencionadas, hay antimicrobianos que pueden tener comportamiento mixto; es por esto, que para fármacos como los aminoglucósidos, quinolonas, glucopéptidos o daptomicina, se puede considerar su actividad como dependiente de Cmax / CIM o de ABC/CIM ${ }^{31}$.

A continuación se presentan los índices $\mathrm{PK} / \mathrm{PD}$ para los antimicrobianos utilizados comúnmente en el paciente con neutropenia febril post-quimioterapia.

$P K / P D$ de los $\beta$-lactámicos en pacientes con neutropenia febril post-quimioterapia:

Los experimentos en animales han demostrado que su actividad es lenta y casi por completo dependiente de tiempo. La máxima eficacia se logra cuando la concentración se mantiene 4 a 5 veces por encima de la CIM (concentraciones mayores no ofrecen beneficio adicional). En modelos animales de infección, se encontró que no es necesario mantener estas concentraciones durante el $100 \%$ del intervalo de dosificación, para conseguir efectos antibacterianos significativos. En estos estudios se encontró mortalidad de casi $100 \%$ cuando el T > CIM fue menor o igual al 20\% del intervalo de dosificación, en contraste con tasas de supervivencia de 90 a $100 \%$ cuando las concentraciones del antimicrobiano estuvieron por encima de la CIM entre 40 y $50 \%$ del intervalo de dosificación; con T > CIM de 30 a 40\%, se encontró efecto bacteriostático ${ }^{(34-37)}$.

Los estudios in vitro sugieren que el tiempo sobre la CIM se debe mantener por el 50\% del intervalo entre dosis para las penicilinas, 60 a $70 \%$ para las cefalosporinas y $40 \%$ para los carbapenems; estos resultados reflejan la variación en la tasa de erradicación, que es más rápida para los carbapenémicos, y más lenta para las cefalosporinas $^{30,38}$. Lo cual, como se mencionó, se puede lograr usando dosificaciones más frecuentes, infusiones prolongadas o continuas. En el estudio retrospectivo de Lodise y cols., se encontró reducción de la mortalidad (12,2 vs 
$31,6 \% ; \mathrm{P}=0,04)$ y disminución de la estancia hospitalaria ( 21 vs. 38 días; $\mathrm{P}=0,02$ ) con el uso de infusiones prolongadas (infusión de 4 horas cada 8 horas) de piperacilina / tazobactam comparada con administración intermitente en pacientes críticamente enfermos con infección por $P$. aeruginosa ${ }^{39}$. En otro estudio se encontró que una dosis $25 \%$ más baja de piperacilina/tazobactam administrada en infusión continua mantuvo concentraciones valle más altas en comparación con la dosificación en bolos ${ }^{40}$.

En los pacientes con neutropenia febril se han encontrado importantes variaciones en la farmacocinética de los b-lactámicos; por ejemplo, en pacientes tratados con ceftazidima se encontró una vida media terminal más corta y un área bajo la curva (ABC) más baja. Aunque no hubo una diferencia significativa, el volumen de distribución tuvo tendencia a ser mayor comparado con personas sanas $^{41}$. En pacientes tratados con imipenem se encontró vida media más larga, menor depuración y volúmenes de distribución más grandes que en voluntarios sanos. Estos cambios se asociaron con mayor edad y la gravedad de la enfermedad ${ }^{42}$. En pacientes tratados con meropenem se encontró aumento en el volumen de distribución y en la eliminación no renal en los pacientes con neutropenia febril comparado con sujetos sanos ${ }^{43}$.

Se han realizado otros estudios que evalúan la probabilidad de lograr las metas PK/PD con diferentes esquemas de dosificación. En el caso de imipenem, Lamoth y cols., usando el programa para análisis de farmacocinética poblacional NONMEM (acrónimo para non-linear mixed effects modeling), encontraron que el régimen de $2 \mathrm{gr} /$ día logró el cubrimiento de los microorganosmos más comunes durante el intervalo de dosificación sólo en 53\% de los pacientes. La meta se logró en $90 \%$ de pacientes con dosis de $3 \mathrm{gr} / \mathrm{día}^{44}$. Para meropenem, en el estudio de Lee y cols., en 1.000 pacientes simulados tratados con meropenem 0,5 gr o 1 gr cada 8 horas las proporciones de pacientes con tiempos superiores a la CIM (T > CIM) menor de $40 \%$ del intervalo de dosis fue 46,3 y $39,5 \%$ para P.aeruginosa y 5,8 y $5,6 \%$ para E. coli, respectivamente ${ }^{45}$ (Figura 1).

En este estudio, para calcular el T > CIM, se utilizó la siguiente ecuación, basada en el modelo de eliminación lineal monocompartimental:

\section{$\mathrm{T}>\mathrm{CIM}=\underline{\mathrm{Ln}(\text { Concentración en estado estacionario/CIM })}$ (Depuración/Volumen de distribución)}

En un estudio retrospectivo, se encontraron desenlaces clínicos similares (tiempo para defervescencia de la fiebre, necesidad de antimicrobianos adicionales, duración de la terapia, mortalidad y efectos adversos) al comparar la dosis de meropenem de 0,5 g cada 6 horas con la dosificación tradicional de $1 \mathrm{~g}$ cada 8 horas y con la dosificación habitual de imipenem/cilastatina (500 mg cada 6 horas) en adultos con neutropenia febril ${ }^{46}$. En otro estudio, también retrospectivo, se encontró respuesta clínica del $80 \%$ en pacientes tratados con meropenem cuando el $\mathrm{T}$ $>$ CIM se mantuvo durante el $75 \%$ del intervalo de dosis concluyendo que la dosificación de $500 \mathrm{mg}$ cada 6 horas puede ser comparable con la dosis de 1 gr cada 8 horas $^{47}$.

\section{PK/PD y glucopéptidos}

El estudio de Hyatt y cols., demostró que los pacientes tratados con vancomicina como monoterapia para infecciones por enterococos que lograron $\mathrm{ABC} / \mathrm{CIM}<125$ tenían mayor probabilidad de falla terapéutica y selección de cepas resistentes de $E$. faecium $^{48}$. En infección por $S$. aureus resistente a meticilina (SARM) Moise-Broder y cols., establecieron que el ABC/CIM que predecía desenlaces clínicos y bacteriológicos favorables es superior a $350^{49}$.

Con el objetivo de simplificar el estudio de la farmacocinética, se diseñaron modelos de funcionamiento basados fundamentalmente en la consideración del organismo como compartimentos relacionados entre sí. De estos modelos, los más utilizados son el monocompartimental y el bi-compartimental. En el modelo de compartimiento único, se considera que un medicamento luego de entrar al torrente sanguíneo, se distribuye en un solo compartimiento total y luego es eliminado a una tasa o constante de eliminación determinada. En el modelo bi-compartimental, se considera que un medicamento luego de haber entrado al torrente sanguíneo, se distribuye en un compartimiento central, de allí pasa a un segundo compartimiento periférico, y luego debe retornar al central para poder ser eliminado. En este caso se habla de una fase $\alpha$ predominando el proceso de distribución y una

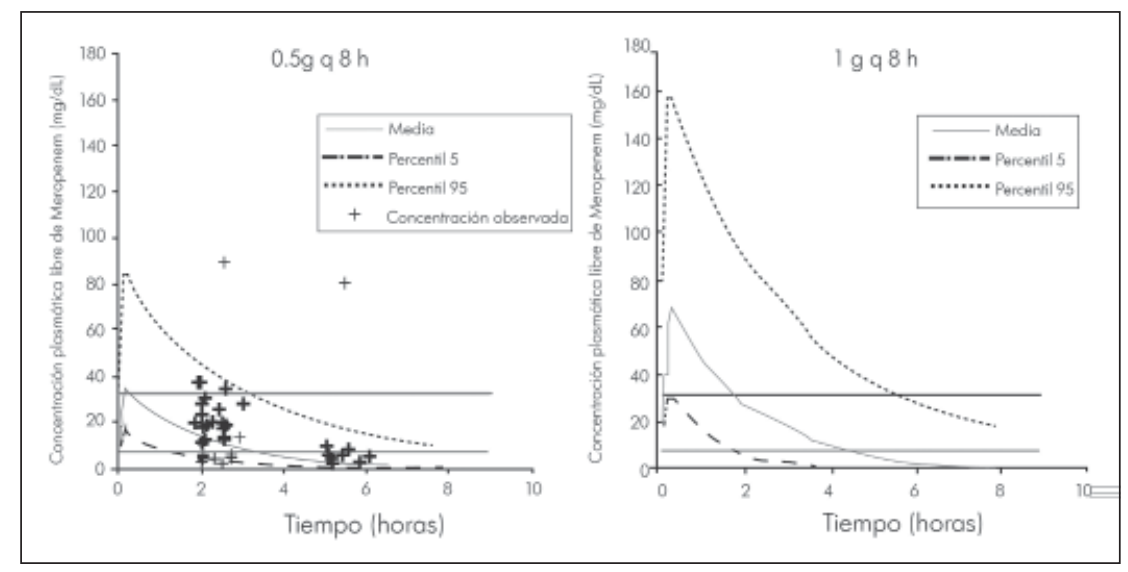

Figura 1. Concentraciones plasmáticas simuladas de meropenem tras la administración de 0,5 gr o 1 gr cada 8 horas. Las $\mathrm{CIM}_{50}$ y $\mathrm{CIM}_{90}$ son $8 \mathrm{mg} / \mathrm{mL}$ y $32 \mathrm{mg} / \mathrm{mL}$, respectivamente, para 43 aislados de P. aeruginosa del Centro Católico de Trasplante Hematopoyético de Corea. Las cruces corresponden a las CIM de los aislados. Gráfica reproducida con la autorización de los autores: Lee y cols. Population pharmacokinetics of meropenem in febrile neutropenic patients in Korea. Intern J Antimicrob Agents 2006; 28: 333-9. 
fase $\beta$ donde predomina el proceso de eliminación ${ }^{50}$. Para describir el comportamiento farmacocinético de vancomicina, en la mayoría de estudios se utiliza el modelo bi-compartimental ${ }^{51,52,55}$.

En pacientes con neutropenia febril se ha encontrado aumento de tres veces en el volumen de distribución y disminución de tres veces en la vida media $\beta$ en comparación con sujetos normales ${ }^{51}$. En pacientes con neoplasias hematológicas se encontraron mayores tasas de depuración y volumen de distribución; el aumento en el volumen de distribución se asoció con el peso corporal total, mientras que la función renal y el diagnóstico de leucemia mieloide aguda influyeron en la depuración. Las características clínicas de los pacientes como la gravedad, tiempo post-quimioterapia, presencia de neutropenia o trasplante de precursores hematopoyéticos no tuvieron correlación con la disposición de vancomicina ${ }^{52}$.

En el caso de teicoplanina, se encontró un aumento significativo en la tasa de eliminación y vida media más corta en pacientes neutropénicos comparado con voluntarios sanos. La variabilidad entre los individuos en las tasas de eliminación fue explicada por la variabilidad en la depuración de creatinina; la variabilidad en los volúmenes de distribución se asoció con la edad y el recuento de leucocitos ${ }^{53}$.

La dosificación empírica de vancomicina basada en la depuración de creatinina debe ir seguida del monitoreo de la concentración mínima $\left(\mathrm{C}_{\min }\right.$ : mantener $15 \mathrm{mg} / \mathrm{L}$ a $20 \mathrm{mg} / \mathrm{L}$ ), también conocido como monitoreo de seguridad, en los pacientes adultos con elevado riesgo de nefrotoxicidad (co-administración de otros nefrotóxicos como aminoglucósidos o anfotericina B), en pacientes con función renal inestable y en quienes reciben tratamiento por periodos prolongados ${ }^{54}$.

Según los análisis PK/PD se asoció la dosificación habitual de vancomicina con un $33 \%$ de riesgo de no lograr las metas farmacodinámicas en infecciones por $S$. aureus en pacientes críticamente enfermos ${ }^{55}$.

\section{PK/PD y quinolonas:}

Presentan actividad dependiente de tiempo y concentración. Se sugiere que la relación entre la concentración máxima $\left(\mathrm{C}_{\max }\right)$ y $\mathrm{CIM}\left(\mathrm{C}_{\max } / \mathrm{CIM}\right)$ de 10 para la ciprofloxacina es crítica para predecir erradicación bacteriana ${ }^{56}$. Otros estudios han demostrado que el ABC/CIM debe ser mayor de 100 a 125 para una adecuada respuesta clínica en infecciones por bacilos gramnegativos, mientras que las cocáceas grampositivas requieren $\mathrm{ABC} / \mathrm{CIM}$ superiores a $30^{38}$.

La dosificación inapropiadamente baja de ciprofloxacina se ha asociado con emergencia de cepas resistentes de enterococos, Pseudomonas spp y SARM. Las nuevas fluoroquinolonas tienen un elevado volumen de distribución con penetración a casi todos los tejidos. De hecho, 60 a
$75 \%$ del medicamento en el cuerpo en cualquier momento se puede encontrar a nivel intracelular lo cual contribuye a una menor excreción renal y mayor vida media ${ }^{10}$. El volumen de distribución se afecta mínimamente en los pacientes críticamente enfermos, aunque levofloxacina requiere aumento de la dosis por disminución de la vida media $^{57}$.

En un estudio farmacocinético realizado en siete pacientes que recibían levofloxacina como profilaxis para neutropenia febril, se encontró depuración de $5,8 \mathrm{~L} / \mathrm{hr}$, Cmax de $3,4 \mathrm{mg} / \mathrm{mL}$ con administración de $200 \mathrm{mg}$ cada 12 horas. Posterior a esto se simuló el comportamiento de dosis diaria de $500 \mathrm{mg}$ encontrando una concentración de $8,54 \mathrm{mg} / \mathrm{mL}$ con lo cual los autores concluyeron que la dosis más efectiva como profilaxis es de $500 \mathrm{mg} /$ día $^{58}$.

\section{PK/PD y aminoglucósidos}

Muestran características dependientes de concentración, tienen efecto post-antibiótico significativo que puede evitar la proliferación bacteriana por periodos prolongados mientras las concentraciones del medicamento caen por debajo de la $\mathrm{CIM}^{59}$. Se recomienda mantener una relación de $\mathrm{C}_{\max } / \mathrm{CIM}$ superior a 10 para una eficacia óptima con dosificación a intervalos prolongados (una vez al día), idealmente monitorizando la $\mathrm{C}_{\text {max }}$ en los pacientes críticamente enfermos, buscando una $\mathrm{C}_{\min }$ muy baja para minimizar la toxicidad ${ }^{38}$.

En pacientes con neoplasias hematológicas se ha encontrado un aumento importante en el volumen de distribución de amikacina comparado con personas sanas ${ }^{60}$. Aparte del peso corporal y la función renal, la leucemia mieloide aguda y la hipoalbuminemia se relacionan con la variabilidad ente los individuos en la farmacocinética de amikacina ${ }^{61}$. En el estudio de Zeitany, en el cual se compararon pacientes con neoplasias hematológicas con controles sanos, se encontraron diferencias significativas, con aumento en el volumen de distribución y la depuración en los pacientes con neoplasias hematológicas. En este mismo estudio, el porcentaje de blastos en la médula en el momento del diagnóstico en pacientes con leucemia aguda, se correlacionó significativamente con aumento en la depuración; los pacientes con linfomas en estadío IV también tuvieron un aumento significativo de la depuración, comparado con pacientes en estadíos más bajos. Ni la presencia de fiebre, ni el recuento de leucocitos, ni la quimioterapia tuvieron efecto significativo en la cinética de los aminoglucósidos. La dosis promedio de amikacina requerida para mantener concentraciones pico séricas por encima de $20 \mathrm{mg} / \mathrm{mL}$ en pacientes con neoplasias hematológicas fue de $27,5 \mathrm{mg} / \mathrm{kg} /$ día. Basado en esto, los autores sugieren para los pacientes con neoplasias hematológicas el uso de amikacina 7,5 mg/ $\mathrm{kg}$ a $10 \mathrm{mg} /$ $\mathrm{kg}$ por dosis cada 8 horas o $2 \mathrm{mg} / \mathrm{kg}$ a $2,5 \mathrm{mg} / \mathrm{kg}$ por dosis cada 8 horas de gentamicina ${ }^{62}$. Sin embargo, en el 
estudio realizado posteriormente por Tod y cols., en el que se comparó la cinética de la amikacina entre dosis de $7,5 \mathrm{mg} / \mathrm{kg}$ cada 12 horas o $20 \mathrm{mg} / \mathrm{kg}$ /día no se encontró diferencia en los parámetros cinéticos. La eliminación de amikacina solo se correlacionó con la depuración de creatinina o sus covariables (sexo, edad, peso y creatinina sérica). Con estos hallazgos, estos autores proponen que la dosificación de amikacina sea de $20 \mathrm{mg} / \mathrm{kg}$ /día para pacientes con función renal normal (depuración de creatinina de $80 \mathrm{ml} / \mathrm{min}$ a $130 \mathrm{ml} / \mathrm{min}$ ) mientras que para pacientes con alteración grave de la función renal se recomiendan dosis de $17 \mathrm{mg} / \mathrm{kg}$ cada 48 horas $^{63}$.

\section{Otros antimicrobianos}

Linezolid es un antibacteriano que pertenece al grupo de las oxazolidinonas. A pesar de ser altamente hidrofílico se distribuye ampliamente en los tejidos, tiene actividad antimicrobiana dependiente de tiempo con efecto post-antibiótico, tanto el $\mathrm{T}>\mathrm{CIM}$ como el ABC/CIM se identificaron como determinantes importantes de su eficacia in vitro e in vivo. Se recomienda mantener una $\mathrm{T}$ $>$ CIM durante 40 a $80 \%$ del intervalo entre dosis y ABC/ CIM entre 48 y 147 para lograr el efecto bacteriostático ${ }^{38}$. En un estudio realizado en pacientes con neutropenia y cáncer no se encontraron diferencias estadísticamente significativas en cuanto a los parámetros farmacocinéticos en los pacientes neutropénicos ${ }^{64}$.

Daptomicina es un lipopéptido cíclico con actividad antimicrobiana dependiente de concentración, su eficacia se correlaciona con Cmax/CIM y ABC/CIM. Se realizó un estudio, en el que se evaluó el comportamiento farmacocinético en pacientes adultos con cáncer y neutropenia febril en el cual se encontró disminución de la Cmax y del ABC, con aumento del volumen de distribución y la depuración al compararlo con adultos sanos ${ }^{65}$.

En la Tabla 2 se resumen las principales variaciones identificadas en diferentes estudios en la PK de los antimicrobianos utilizados en el tratamiento de la neutropenia febril posquimioterapia.

Las alteraciones en la farmacocinética de los antibbacterianos (principalmente los hidrofílicos) en pacientes con neutropenia febril, principalmente consisten en variaciones en el volumen de distribución, la depuración renal y no renal de los mismos. Esto a su vez puede alterar las concentraciones plasmáticas y potencialmente comprometer su eficacia.

\section{Conclusiones}

La neutropenia febril es una complicación frecuente de la terapia antineoplásica que se sigue asociando a altas tasas de mortalidad. Entre los factores que se pueden asociar con fracaso terapéutico están las concentraciones inapropiadas de los antimicrobianos. Teniendo en cuenta que los ensayos clínicos han demostrado que es posible optimizar la formulación de los antimicrobianos con el uso de los parámetros $\mathrm{PK} / \mathrm{PD}$ y que los pacientes con cáncer y neutropenia febril presentan variaciones en los parámetros cinéticos de los antimicrobianos, es posible que al conocer el comportamiento de estos parámetros en nuestros pacientes, se pueda mejorar la dosificación de la antibioterapia para el manejo de la neutropenia febril, con lo que se podrían a su vez mejorar los desenlaces clínicos, disminuir la mortalidad, la toxicidad medicamentosa, el desarrollo de resistencia bacteriana e incluso se podrían reducir las dosis diarias de antimicrobianos y el tiempo de estancia hospitalaria.

Sin embargo, se debe tener presente que la mayoría de estudios en esta área, han sido diseñados para evaluar los cambios en la farmacocinética de los antimicrobianos, que ocurren en los pacientes con neutropenia febril en comparación con adultos sanos; los pocos estudios en los que se ha evaluado la correlación de los parámetros $\mathrm{PK} / \mathrm{PD}$ con desenlaces clínicos son retrospectivos. Hasta la fecha no disponemos de estudios prospectivos en adultos, que respondan si la optimización de la terapia antimicrobiana con los parámetros PK/PD se asocia con mejores desenlaces clínicos. Se requiere mayor número de estudios clínicos, que validen estos supuestos en este grupo específico de pacientes y mayor información local (microorganismos, CIM, cinética de antimicrobianos, etc.) para hacer un ajuste individualizado de la terapia antimicrobiana.

Tabla 2. Variaciones en la farmacocinética de los antibióticos en pacientes con neutropenia febril

\begin{tabular}{|c|c|c|c|c|}
\hline Grupo de antimicrobianos & Volumen de distribución & Cmax & Depuración & Referencias \\
\hline$\beta$ - Lactámicos & & & Normal o disminuida & $41,42,43$ \\
\hline Glucopéptidos & & & Normal, disminuida o aumentada & $51,52,53$ \\
\hline Quinolonas & Normal & Normal & Normal o disminuida & 57 \\
\hline Aminoglucósidos & & & Normal o aumentada & $60,61,62,63$ \\
\hline Oxazolidinonas & Normal & Normal & Normal & 64 \\
\hline Daptomicina & & & Aumentada & 65 \\
\hline
\end{tabular}




\section{Resumen}

La neutropenia febril es una complicación grave de la terapia antineoplásica que se presenta más frecuentemente en pacientes con neoplasias hematológicas, asociada a tasas elevadas de mortalidad. Uno de los factores descritos como causa de fracasos terapéuticos de la terapia antimicrobiana es la inadecuada concentración tisular de los antimicrobianos que a su vez se correlaciona con bajas concentraciones en el líquido intersticial en el caso de los fármacos hidrofílicos. En pacientes críticamente enfermos se puede presentar acumulación compartimental de líquidos que a su vez se puede asociar con aumento en el volumen de distribución de los medicamentos o alteraciones en la depuración de los mismos. Se revisan los parámetros farmacocinéticos y farmacodinámicos de los antimicrobianos que pueden ser usados como herramienta para optimizar la eficacia de la terapia antiinfecciosa en busca de disminuir la tasa de fracasos y la selección de cepas resistentes.

\section{Referencias}

1.- Hughes W, Armstrong D,Bodey G, Bow E, Brown A, Calandra T, et al. 2002 Guidelines for the use of antimicrobial agents in neutropenic patients with cancer. Clin Infect Dis 2002; 34: 730-51.

2.- $\quad$ Sculier J P, Weerts D, Klastersky J. Causes of death in granulocytopenic cancer patients receiving empiric antibiotic therapy. Eur $\mathrm{J}$ Cancer Clin Oncol; 20 (1): 55-60.

3.- Rossi C, Klastersky J. Initial antibiotic empirical therapy for neutropenic fever: analysis of the causes of death. Support Care Cancer 1996; 4 (3): 207-12

4.- Muñoz O, Rodelo A, Carvajal J, González J, Jaimes F. Características clínicas y microbiológicas de los pacientes neutropénicos febriles con neoplasias hematológicas. Iatreia 2008; 21 (1): s9.

5.- Viscoli C, Varnier O, Machetti M L. Infections in patients with febrile neutropenia: epidemiology, microbiology, and risk stratification. Clin Infect Dis 2005; 40: S240-5.

6.- Hann I, Viscoli C, Paesmans M, Gaya H, Glauser M. A comparison of outcome from febrile neutropenic episodes in children compared with adults: results from four EORTC studies. International Antimicrobial Therapy Cooperative Group (IATCG) of the European Organization for Research and Treatment of Cancer (EORTC). Br J Haematol 1997; 99 (3): 580-8.

7.- Meckler G, Lindemulder S. Fever and neutropenia in pediatric patients with cancer. Emerg Med Clin North Am 2009; 27 (3): 525-44.

8.- Hakim H, Flynn P M, Knapp K M, Srivastava D K, Gaur A H. Etiology and clinical course of febrile neutropenia in children with cancer. $\mathrm{J}$ Pediatr Hematol Oncol 2009; 31 (9): 623-9.

9.- Badiei Z, Khalesi M, Alami M H, Kianifar H R, Banihashem A, Farhangi H, et al. Risk factors associated with life-threatening infections in children with febrile neutropenia: a data mining approach. J Pediatr Hematol Oncol 2011; 33 (1): e9-e12.

10.- Scaglione F, Paraboni L. Pharmacokinetics/ pharmacodynamics of antibacterials in the Intensive Care Unit: setting appropriate dosing regimens. Int J Antimicrob Agents 2008; 32: 294-301.

11.- Lortholary O, Lefort A, Tod M, Chomat A, Darras-Joly C, Cordonnier C. Pharmacodynamics and pharmacokinetics of antibacterial drugs in the management of febrile neutropenia. Lancet Infect Dis 2008; 8: 612-20.

12.- Rolston K. Challenges in the treatment of infections caused by gram-positive and gramnegative bacteria in patients with cancer and neutropenia. Clin Infect Dis 2005; 40: S246-52.

13.- Padrón N, Menéndez S. Infecciones en el paciente neutropénico con cáncer. Rev Panam Infectol 2006; 8 (3): 24-34.

14.- Sipsas N, Bodey G, Kontoyiannis D. Perspectives for the management of febrile neutropenic patients with cancer in the $21 \mathrm{st}$ Century. Cancer 2005; 103 (6): 1103-13.

15.- Zinner SH. Changing epidemiology of infections in patients with neutropenia and cancer: emphasis on gram-positive and resistant bacteria. Clin Infect Dis 1999; 29 (3): 490-4.

16.- Yadegarynia D, Tarrand J, Raad I, Rolston K. Current spectrum of bacterial infections in patients with cancer. Clin Infect Dis 2003; 37: 1144-5.

17.- Cortés J, Cuervo S, Arroyo P, Quevedo R. Hallazgos microbiológicos en pacientes con neutropenia febril. Rev Colomb Cancerol 2003; 7 (4): 5-11.

18.- Figuera M, Carballo M, Silva M, Figueredo A, Avilán J. Aislamientos microbiológicos en pacientes con neutropenia febril y neoplasias hematológicas. Rev Esp Quimioter 2006; 19 (3): 247-51.

19.- Rabagliati R, Fuentes G, Orellana E, Oporto J, Domínguez I, Benítez R, et al. Etiología de episodios de neutropenia febril en pacientes adultos con cáncer hematológico y de órganos sólidos en el Hospital Clínico Universidad Católica, Santiago-Chile. Rev Chilena Infectol 2009; 26 (2): 106-13.

20.- Bernal E, Cuervo S, Arroyo C, Ramos P. Microbiología y susceptibilidad antimicrobiana en neutropenia febril en el Instituto Nacional de Cancerología (INC) de Bogotá. Infectio 2008;
12 (Supl 1): 1-2.

21.- Segal B, Baden L, Brown A, Casper C, Dunnerke E, Freifeld A, et al. NCCN Prevention and treatment of cáncer related infections. Practice guidelines in Oncology v.1.2008.

22.- Rodríguez G, Gobernado M, Gomis M, Mensa J, Picazo J, Prieto J et al. Guía clínica para la evaluación y el tratamiento del paciente neutropénico con fiebre. Rev Esp Quimoter 2001; 14: 75-83

23.- Link H, Bohme A, Cornely OA, Hoffken K, Kellner O, Kern W, et al. Antimicrobial therapy of unexplained fever in neutropenic patients-Guidelines of the Infectious Diseases Working Party (AGIHO) of the German Society of Hematology and Oncology (DGHO), Study Group Interventional Therapy of Unexplained Fever, ArbeitsgemeinschaftSupportivmassnahmen in der Onkologie (ASO) of the Deutsche Krebsgesellschaft (DKGGerman Cancer Society). Ann Hematol 2003; 82(Suppl 2): S105-17.

24.- Paul M, Soares-Weiser K,Leibovici L. $\beta$-lactam monotherapy versus $\beta$-lactam-aminoglycoside combination therapy for fever with neutropenia: systematic review and meta-analysis. Br Med J 2003; 326: 1111.

25.- Furno P, Bucaneve G, Del Favero A. Monotherapy or aminoglycoside-containing combinations for empirical antibiotic treatment of febrile neutropenic patients: a meta-analysis. Lancet Infect Dis 2002; 2: 231-42

26.- Power B M, Forbes A M, van Heerden P V, Ilett K F. Pharmacokinetics of drugs used in critically ill adults. Clin Pharmacokinet 1998; 34 (1): $25-56$

27.- Jellett L B, Heazlewood V J. Pharmacokinetics in acute illness. Med J Aust 1990; 153: 534-41.

28.- Vrhovac B, Sarapa N, Bakran I, Huic M, Macolic-Sarinic V, Francetic I, et al. Pharmacokinetic changes in patients with oedema. Clin Pharmacokinet 1995; 28: 405-18.

29.- Quintiliani R, Quintiliani, R Jr. Pharmacokinetics / pharmacodynamics for critical care clinicians. Crit Care Clin 2008; 24 : 335-48.

30.- Craig W. Pharmacokinetic/pharmacodynamic 
parameters: rationale for antibacterial dosing of mice and men. Clin Infect Dis 1998; 26: 1-12.

31.- Nightingale C, Ambrose P, Drusano G, Murakawa T. Antimicrobial Pharmacodynamics in Theory and Clinical Practice. Second Edition. 2007.

32.- Rybak M. Pharmacodynamics: Relation to antimicrobial resistance. Amer J Med 2006; 119 (6A): S37-S44.

33.- Levison M, Levison J. Pharmacokinetics and pharmacodynamics of antibacterial agents. Infect Dis Clin N Am 2009; 23: 791-815.

34.- Craig WA. Interrelationship between pharmacokinetics and pharmacodynamics in determining dosage regimens for broadspectrum cephalosporins. Diagn Microbiol Infect Dis 1995; 22: 89-96.

35.- Vogelman B, Gudmundsson S, Leggett J, Turnidge J, Ebert S, Craig W A. Correlation of antimicrobial pharmacokinetic parameters with therapeutic efficacy in an animal model. J Infect Dis 1988; 158: 831-47.

36.- Leggett J E, Fantin B, Ebert S, Totsuka K, Vogelman B, Calame W, et al. Comparative antibiotic dose-effect relations at several dosing intervals in murine pneumonitis and thighinfection models. J Infect Dis 1989; 159: 281-92.

37.- Roosendaal R, Bakker-Woudenberg I A J M, van den Berg J C, Michel M F. Therapeutic efficacy of continuous versus intermittent administration of ceftazidime in an experimental Klebsiella pneumoniae pneumonia in rats. J Infect Dis 1985; 152: 373-8.

38.- Roberts J, Kruger P, Paterson D, Lipman J. Antibiotic resistance-What's dosing got to do with it? Crit Care Med 2008 Aug; 36(8): 243340 .

39.- Lodise T, Lomaestro B, Drusano G. Piperacillin-tazobactam for Pseudomonas aeruginosa infection: Clinical implications of an extended- infusion dosing strategy. Clin Infect Dis 2007; 44: 357-63.

40.- Roberts J, Roberts M, Robertson T, Dalley A, Lipman J. Piperacillin penetration into tissue of critically ill patients with sepsis-Bolus versus continuous administration? Crit Care Med 2009; 37 (3): 926-33.

41.- Nyhlen A, Ljungberg B, Nilsson-Ehle I. Pharmacokinetics of ceftazidime in febrile neutropenic patients. Scand J Infect Dis 2001; 33: 222-6.

42.- Drusano G, Plaisance K, Forrest A, Bustamante C, Devlin A, Standiford H, et al. Steady-state pharmacokinetics of imipenem in febrile neutropenic cancer patients. Antimicrob Agents Chemother 1987; 1420-2.

43.- Nyhlen A, Ljungberg B, Nilsson-Ehle I.
Pharmacokinetics of meropenem in febrile neutropenic patients. Swedish study group. Eur J Clin Microbiol Infect Dis 1997; 16 (11): 797-802.

44.- Lamoth F, Buclin T, Csajka C, Pascual A, Calandra T, Marchetti O. Reassessment of recommended imipenem doses in febrile neutropenic patients with hematological malignancies. Antimicrob Agents Chemother 2009; 53 (2): 785-7.

45.- Lee D, Choi S, Shin W, Lahb H, Yimb D. Population pharmacokinetics of meropenem in febrile neutropenic patients in Korea. Int $\mathrm{J}$ Antimicrob Agents 2006; 28: 333-9.

46.- Arnold H M, McKinnon P S, Augustin K M, Hladnik L M, Casabar E, Reichley R M, et al. Assesment of an alternative meropenem dosing strategy compared with imipenem-cilastatin or traditional meropenem dosing after cefepime failure or intolerance in adults with neutropenic fever. Pharmacotherapy 2009; 29 (8): 914-23.

47.- Ariano R, Nyhlén A. Pharmacokinetics and Pharmacodynamics of meropenem in febrile neutropenic patients with bacteremia. Ann Pharmacother 2005; 39 (1): 32-8.

48.- Hyatt J M, McKinnon P S, Zimmer G S, Schentag J J. The importance of pharmacokinetic/pharmacodynamic surrogate markers to outcome. Focus on antibacterial agents. Clin pharmacokinet 1995; 28 (2): 14360.

49.- Moise-Broder P A, Forrest A, Birmingham M C, Schentag J J. Pharmacodynamics of vancomycin and other antimicrobials in patients with Staphylococcus aureus lower respiratory tract infections. Clin Pharmacokinet 2004; 43 (13): 925-42.

50.- Birkett D. Pharmacokinetics made easy. McGraw Hill Company Inc. 2002.

51.- Le Normand Y, Milpied N, Kergueris M F, Harousseau J L. Pharmacokinetic parameters of vancomycin for therapeutic regimens in neutropenic adult patients. Int J Biomed Comput 1994; 36 (1-2): 121-5.

52.- Buelga D, Fernández M, Herrera E, DomínguezGil A, GarcíaM. Population pharmacokinetic analysis of vancomycin in patients with hematological malignancies. Antimicrob Agents Chemother 2005; 49 (12): 4934-41

53.- Lortholary O, Tod M, Rizzo N, Padoin C, Biard O, Casassus P et al. Population pharmacokinetic study of teicoplanin in severely neutropenic patients. Antimicrob Agents Chemother 1996; 40 (5): 1242-7.

54.- Rybak M, Lomaestro B, Rotschafer J, Moellering R, Craig W, Billeter M. Therapeutic monitoring of vancomycin in adult patients: A consensus review of the American Society of Health-System Pharmacists, the Infectious Diseases Society of America, and the Society of Infectious Diseases Pharmacists. Am J HealthSyst Pharm. 2009; 66: 82-98.

55.- Fernández de Gatta M, Revilla N, Calvo Alfonso M, Sánchez Navarro A. Pharmacokinetic/pharmacodynamic analysis of vancomycin in ICU patients. Intensive Care Med 2007; 33: 279-85.

56.- Drusano G L, Johnson D E, Rosen M, Standiford H C. Pharmacodynamics of a fluoroquinolone antimicrobial agent in a neutropenic rat model of Pseudomonas sepsis. Antimicrob Agents Chemother 1993; 37:483-90.

57.- Roberts J, Lipman J. Pharmacokinetic issues for antibiotics in the critically ill patient. Crit Care Med 2009; 37 (3): 840-51.

58.- Nomura K, Fujimoto Y, Morimoto Y, Kanbayashi Y, Matsumoto Y and Taniwaki M. Population pharmacokinetics of levofloxacin as prophylaxis for febrile neutropenia. Inter Med 2008; 47 (5): 375-8.

59.- Hatala R, Dinh T T, Cook D L. Single daily dosing of aminoglycosides in immunocompromised adults: a systematic review. Clin Infect Dis 1997; 24 (5): 810-5.

60.- Kaojarern S, Maoleekoonpairoj S, Atichartakarna V. Pharmacokinetics of amikacin in hematologic malignancies. Antimicrob Agents Chemother 1989; 33 (8): 1406-8.

61.- Romano S, Fdez de Gatta M M, Calvo M V, Caballero D, Domínguez Gil D, Lanao J M. Population pharmacokinetics of amikacin in patients with haematological malignancies. J Antimicrob Chemother 1999; 44 (2): 235 42.

62.- Zeitany R, El Saghir N, Santhosh-Kumar C, Sigmon M. Increased aminoglycoside dosage requirements in hematologic malignancy. Antimicrob Agents Chemother 1990; 34 (5): 702-8.

63.- Tod M, Lortholary O, Seytre D, Semaoun R, Uzzan B, Guillevin L, et al. Population pharmacokinetic study of amikacin administered once or twice daily to febrile, severely neutropenic adults. Antimicrob Agents Chemother 1998; 42 (4): 849-56.

64.- Smith P, Birmingham M, Noskin G, Meaghe A, Forrest A, Rayner C, Schentag J. Safety, efficacy and pharmacokinetics of linezolid for treatment of resistant Gram-positive infections in cancer patients with neutropenia. Ann Oncol 2003; 14 (5): 795-801.

65.- Bubalo J, Munar M, Cherala G, Hayes-Lattin B, Maziarz R. Daptomycin pharmacokinetics in adult oncology patients with neutropenic fever. Antimicrob Agents Chemother 2009; 53 (2): 428-34. 